\title{
Persiste la incertidumbre en la relación entre la hipertensión arterial y el riesgo de epistaxis
}

\author{
Uncertainty persists on the relationship between the arterial hypertension and the risk of epistaxis
}

\section{Comentado de:}

Byun $H$, et al JAMA Otolaryngol Head Neck Surg. 2021;147(1):34-40. PMID: $32910190^{1}$

\section{Objetivo}

Evaluar si en pacientes con hipertensión arterial (HTA) el riesgo de desarrollar epistaxis y su severidad es mayor que en la población general.

\section{Diseño, lugar y pacientes}

Se diseñó un estudio de cohorte retrospectiva utilizando la base de datos oficial de salud de Corea del Sur: The National Health Insurance Service-National Sample Cohort, una cohorte sistematizada aleatorizada estratificada realizada por el sistema de salud nacional (KNHIS, por sus iniciales en inglés), que ofrece a investigadores de salud pública y responsables de realizar políticas sanitarias información médica fiel y útil sobre los ciudadanos del país. Esta cohorte representa el 2,2 \% (1.025.340 personas) de la población total elegible en 2002 , a la que se le realizó seguimiento hasta diciembre de 2015. Cuenta con información de cada participante sobre datos personales, vivienda, consultas al sistema de salud, diagnósticos médicos, medicación habitual, tratamientos y terapéuticas, y muerte ${ }^{2}$.

\section{Criterios de exclusión}

Pacientes que recibieran tratamiento anticoagulante; tuvieran diagnóstico de coagulopatía o enfermedad hereditaria (telangiectasia hemorrágica hereditaria o púrpura trombocitopénica idiopática), neoplasia nasal/paranasal u otra malformación congénita; o hubieran presentado algún traumatismo o cirugía los 30 días previos al evento de epistaxis.

\section{Criterios de selección}

Se incluyeron en el grupo de pacientes con HTA a quienes tenían diagnóstico de HTA esencial, habían consultado al menos tres veces al sistema de salud y estaban bajo tratamiento antihipertensivo. Los pacientes que no cumplieron con estos criterios a lo largo de los 13 años de seguimiento, fueron clasificados en el grupo control.

Se realizó un apareamiento entre el grupo HTA y el grupo control utilizando el puntaje de propensión (estrategia conocida en inglés como propensity score matching) 1:1 según la edad, el sexo, el nivel de ingresos, el lugar de la vivienda, y el Índice de Comorbilidad de Charlson (ICC), que estima la supervivencia a 10 años de acuerdo a la edad del paciente y otros 19 factores de riesgo ${ }^{3}$.

\section{Medición de resultados principales}

Los desenlaces evaluados fueron: la epistaxis, la recurrencia de la epistaxis, el tipo de tratamiento utilizado (conservador o uso de vasoconstrictores locales; cauterización química; taponaje anterior; taponaje posterior; o manejo quirúrgico) y el ámbito donde fue realizada la consulta (departamento de emergencias; centro de atención primaria de salud [CAPS]; centro de referencia ambulatorio).

\section{Resultados principales}

La población del estudio que calificó en el grupo HTA fue de 35.781 participantes, mientras que los controles fueron seleccionados a partir de una población 351.732 personas. Luego de apareamiento 1:1, cada grupo quedó conformado por 35.749 participantes. En el grupo de pacientes con HTA el $57,6 \%$ era de sexo masculino, con una mediana de edad de 52 años (rango intercuartilo 45 a 62 años). No se observaron diferencias entre los grupos en las variables de edad, sexo, tiempo de seguimiento, ingresos, lugar de vivienda, ni mediana del ICC.

Los resultados principales se resumen en la Tabla 1. Al analizar el tipo de tratamiento instaurado en el episodio de epistaxis, el taponaje posterior fue el único que mostró una diferencia estadísticamente significativa con mayor incidencia en el grupo HTA que en el grupo control (razón de probabilidades [OR] 0,5; intervalo de confianza $[I C]$ del $95 \% 0,03$ a 0,26). El grupo control presentó más consultas a los CAPS, mientras que el grupo HTA realizó más consultas al departamento de emergencia (OR 2,69; IC $95 \%$ 1,70 a 4,25 y OR 0,55 ; IC $95 \% 0,40$ a 0,75, respectivamente).

\section{Conclusiones de los autores}

Los autores concluyen que su estudio sugiere que los pacientes con hipertensión tenían un mayor riesgo de epistaxis que requirieron visitas al hospital, principalmente al servicio de urgencias, y necesitaron más procedimientos de taponamiento nasal posterior en comparación con los pacientes sin hipertensión. Recomiendan el asesoramiento médico sobre la epistaxis en las personas con hipertensión, y que debe considerarse la presencia de hipertensión en el manejo de las hemorragias nasales.

\begin{abstract}
Fuente de financiamiento/Conflicto de interés de los autores: Estudio financiado por el Ministerio de Educación de Corea del Sur, a través del Programa de Investigación en Ciencias Básicas de la Fundación Nacional de Investigación. Los autores reportaron no poseer conflictos de interés.
\end{abstract}

Tabla 1. Incidencia de primer episodio de epistaxis y recurrencias en pacientes con hipertensión arterial y en el grupo control. Notas: IC: intervalo de confianza; HTA: hipertensión arterial. *Tasa de incidencia cada 10.000 personas

\begin{tabular}{|l|c|c|c|c|c|}
\hline Desenlaces & $\begin{array}{c}\text { N eventos/ N } \\
\text { expuestos HTA }\end{array}$ & $\begin{array}{c}\text { Tasa de incidencia } \\
\text { grupo HTA* (IC } \\
95 \%)\end{array}$ & $\begin{array}{c}\text { N eventos/ N } \\
\text { expuestos control }\end{array}$ & $\begin{array}{c}\text { Tasa de incidencia } \\
\text { grupo control* } \\
95 \%)\end{array}$ & $\begin{array}{c}\text { Razón de tasas de } \\
\text { incidencia (IC 95\%) }\end{array}$ \\
\hline Epistaxis & $696 / 35.749$ & $\begin{array}{c}32,97(30,57 \text { a } \\
35,51)\end{array}$ & $483 / 35.749$ & $22,76(20,78$ a 24,89) & $1,45(1,29$ a 1,63$)$ \\
\hline $\begin{array}{l}\text { Recurrencia de } \\
\text { epistaxis }\end{array}$ & $42 / 35.749$ & $1,96(1,41$ a 2,65$)$ & $34 / 35.749$ & $1,59(1,10$ a 2,22) & $1,23(0,77$ a 2,00$)$ \\
\hline
\end{tabular}




\section{Comentario}

En este estudio el grupo de pacientes con HTA tuvo 1,45 veces más riesgo de presentar epistaxis que el grupo control. En cuanto a la incidencia de recurrencia de la epistaxis, no pudo establecerse una diferencia estadísticamente significativa, lo que resulta concordante con los resultados de estudios previos que plantean que el riesgo de recurrencia depende del tipo de tratamiento instaurado en primera instancia, si se trata o no de un sangrado posterior, si se pudo localizar o no el vaso sangrante, y no tanto de factores de riesgo predisponentes ${ }^{4}$.

Por último, sugieren que el grupo HTA podría tener epistaxis de mayor severidad por presentar más consultas al departamento de emergencias y por necesitar con mayor frecuencia un taponaje posterior para tratar el sangrado.

Los autores proponen que la presencia de HTA en pacientes con epistaxis debe ser considerada para establecer el manejo y el tratamiento de este factor de riesgo.

En cuanto a las posibles limitaciones de la metodología adoptada por los investigadores, es importante prestar atención a los criterios con que se seleccionaron los pacientes en el grupo HTA: tener diagnóstico de este factor de riesgo, haber consultado al sistema de salud al menos tres veces y recibir tratamiento farmacológico. De esta manera, el grupo HTA queda conformado por pacientes con hipertensión en tratamiento con antihipertensivos. No queda explicitado cuáles son los fármacos utilizados por los participantes, las dosis que reciben, si fuera un único antihipertensivo o una combinación de ellos. Vale la pena entonces preguntarse si la relación entre el factor de riesgo y la epistaxis no se vio condicionada por la exposición a los fármacos, debiendo hablarse entonces de una asociación entre la epistaxis y el tratamiento antihipertensivo, en lugar de las personas con hipertensión.

Tampoco se brinda información sobre la adherencia al tratamiento antihipertensivo o la respuesta a éste, no se explicita si los pacientes tenían o no su presión arterial controlada, ni se informan las cifras tensionales al momento de la consulta.

Una relación distinta que podría plantearse es que, quizás, los pacientes con HTA tienen a consultar con mayor frecuencia a los departamentos de emergencia por el conocimiento de este diagnóstico previo, y es posible que, en ese ámbito, haya más accesibilidad a los tratamientos invasivos, lo que explicaría la mayor incidencia de taponaje posterior. En cambio en los CAPS, ámbito en los que consulta población con menos factores de riesgo, tal vez, la tendencia sea realizar tratamientos más conservadores.

Múltiples estudios previos han relacionado la HTA con las hemorragias nasales ${ }^{5,6}$, aunque los estudios que exploraron específicamente esta relación no pudieron confirmar la asociación entre ambas ${ }^{7,8}$.

Este estudio sugiere que la HTA prolongada contribuye a un riesgo elevado de epistaxis, probablemente debido a sus efectos vasculopáticos $^{8}$. Algunos datos sugieren que la HTA no causa epistaxis pero prolonga los episodios de hemorragia ${ }^{9}$; este punto de vista carece hasta el momento de datos que lo respalden.

\section{Conclusiones de la comentadora}

Los autores se plantean identificar si existe una relación entre la HTA y el desarrollo de epistaxis, su severidad y su terapéutica. Sin embargo, la forma de selección de la población en el grupo HTA nos permite cuestionar si este estudio logra responder esa pregunta. En este sentido, se puede poner en duda si lo que se responde es efectivamente el riesgo de tener epistaxis en pacientes hipertensos, o si en realidad, la relación planteada es entre el riesgo de epistaxis y el tratamiento antihipertensivo.

Con el paso del tiempo y los sucesivos estudios, persiste sin responderse la pregunta original, lo que nos hace repensar la implicancia clínica que tiene contestarla, especialmente si, con los nuevos conocimientos, planteamos que es posible que los valores de presión arterial no estén relacionados con la aparición de la epistaxis. Quizás lo prudente sea continuar promoviendo el control adecuado de la presión arterial sin pensar que cifras altas tengan un rol protagónico en el desarrollo de la epistaxis.

Carolina Kellner [ Servicio de Medicina Familiar y Comunitaria, Hospital Italiano de Buenos Aires. carolina.kellner@hospitalitaliano.org.ar ]

Kellner C. Persiste la incertidumbre en la relación entre la hipertensión arterial y el riesgo de epistaxis . Evid Actual Pract Ambul. $2021 ; 24(3): e 002146$. Available from: https://dx.doi.org/10.51987/EVIDENCIA.V24I4.6967. Comentado de: Byun H, et al. Association of Hypertension With the Risk and Severity of Epistaxis. JAMA Otolaryngol Head Neck Surg. 2021;147(1):34-40. PMID: 32910190. doi:10.1001/jamaoto.2020.2906

\section{Referencias}

1. Byun H, Chung JH, Lee SH, et al. Association of Hypertension With the Risk and Severity of Epistaxis. JAMA Otolaryngol Head Neck Surg. 2021;147(1):34-40. Available from: 10.1001/jamaoto.2020.2906.

2. National Health Insurance Data Sharing Service;. Available from: http://nhiss.nhis.or.kr/ [Last access: 2021-08-12].

3. Charlson Comorbidity Index (CCl);. Available from: https://www.mdcalc.com/charlson-comorbidity-index-cci [Last access: 2021-08-12]

4. Ando Y, limura J, Arai S, et al. Risk factors for recurrent epistaxis: importance of initial treatment. Auris Nasus Larynx. 2014;41(1):41-45. Available from: 10.1016/j.anl.2013.05.004.

5. Abrich V, Brozek A, Boyle TR, et al. Risk factors for recurrent spontaneous epistaxis. Mayo Clin Proc. 2014;89(12):25458126-25458126. Available from: 10.1016/j.mayocp.2014.09.009.

6. Min HJ, Kang H, Choi GJ, et al. Association between Hypertension and Epistaxis: Systematic Review and Meta-analysis. Otolaryngol Head Neck Surg. 2017;157:28742425-28742425. Available from: 10.1177/0194599817721445. 
7. Lubianca-Neto JF, Bredemeier M, Carvalhal EF, et al. A study of the association between epistaxis and the severity of hypertension. Am J Rhinol. 1998;12(4):269-72. Available from: 10.2500/105065898781389985

8. Neto JFL, Fuchs JF, Facco FD, et al. Is epistaxis evidence of end-organ damage in patients with hypertension? Laryngoscope. 1999;109:1111-1116. Available from: 10.1097/00005537-199907000-00019

9. Viehweg TL, Roberson JB, Hudson JW. Epistaxis: diagnosis and treatment. J Oral Maxillofac Surg. 2006;64(3):511-519. Available from: 10.1016/j. joms.2005.11.031. 